\title{
Special Topic: New Materials-Based Luminescence Focusing on Subjects of Chemiluminescence, Fluorescence, Nano Materials and Molecule Probes
}

\author{
Jin-Ming Lin ${ }^{1}$ \\ Published online: 5 December 2017 \\ ๑ The Nonferrous Metals Society of China and Springer Nature Singapore Pte Ltd. 2017
}

The application of new materials for the development of luminescence systems is one of the hottest researches in the area of analytical chemistry. This special issue collected nine papers including electrochemiluminescence (ECL), chemiluminescence (CL) and fluorescence methods enhanced by new materials. As is well-known, ECL is a powerful tool for ultrasensitive detection of a wide range of analytes in chemistry, biology and so on. Apart from traditional metal complex luminophores, nanoluminophores, such as nanocrystals, metal nanoclusters, carbon nanomaterials, have aroused much research interest recently due to its versatility and expected fine properties. That is the focus of this special issue. We accepted six original research papers and two critical reviews in this issue and hope they will provide help to our readers involved in research areas of ECL.

In comparison with organic luminescent probes, quantum dots (QDs) have the advantages of higher photostability, continuous absorption spectra, and size-dependent emission properties. Prof. Guobao Xu prepared a new photoluminescent glucose sensor using inexpensive inorganic boric acid and water-soluble thioglycolic acid-capped $\mathrm{CdTe}$ QDs (TGA@CdTe QDs) in aqueous solution. The luminescent intensities of QDs change linearly with glucose concentrations and display a highly selective response to glucose over other interfering, but biologically important saccharides, amino acids, and common ions. Meanwhile, Prof. Lixia Zhao and Prof. Lianghong Guo reported unprecedented two-step chemiluminescence of polyamine-functionalized carbon dots. Possible CL mechanism of this phenomenon induced by Fenton-like system was investigated based on the fluorescence spectrum, CL emission, FT-IR,

Jin-Ming Lin

jmlin@mail.tsinghua.edu.cn

http://www.linlab-tsinghua-edu.org

1 Department of Chemistry, Tsinghua University, Beijing 100084, China
XPS spectroscopy and the effects of radical scavengers on the CL intensity. Carbon-based material is widely used in chemiluminescence; therefore, Prof. Yi Lv systematically summarized applications of graphitic carbon nitride in review paper titled "Recent Advances in Graphitic Carbon Nitride-Based Chemiluminescence, Cataluminescence and Electrochemiluminescence".

Metal nanoparticles are also important sensor materials. Prof. Chengzhi Huang utilized gold nanoparticles to prepare magnetic bead-based sandwich immunoassay for viral pathogen detection. The analytical sensitivity of the developed immunoassay is over sevenfold higher than that of commercially available RSV ELISA kits and the total assay time was less than $2.5 \mathrm{~h}$ without any pretreatment. Silver nanoparticles (Ag NPs) were deposited on a non-woven fabric used as a surface-enhanced Raman scattering (SERS) substrate for direct detection of pesticide residues in fruits by Prof. Xi Chen. This material could be expected to be a useful tool in rapid on-site test of pesticide residues on fruit surfaces due to its high sensitivity, convenience and nondestructive characteristics.

Prof. Jianzhong Lu prepared streptavidin-modified polystyrene microspheres (PS-SA) and employed it as dual amplification platform for detection of specific DNA sequence by merging the function of HCR and PS-SA amplification. As compared to traditional sandwich type (capture/target/reporter) assays, this dual-amplified strategy for sequence-specific DNA detection showed better specificity, lower detection limit and wider linear response range.

All the above papers are from the view of novel material sensor, Prof. Jin-Ming Lin focuses on gas-solid phase chemiluminescence where $\mathrm{CL}$ is emitted through chemical reactions between gas and solid reactants occurred on the surface of solid matter. In their review, the main classifications including $\mathrm{O}_{3}$-solid, $\mathrm{O}_{2}$-solid, "S-based"-solid, $\mathrm{H}_{2}$-solid and CO-solid CL systems are summarized. 
Finally, we would like to express our sincere thanks to all the contributors to this special issue, and all referees are appreciated for their careful review on the submitted manuscripts. Journal of Analysis and Testing as a new journal will provide an international academic platform for the publication of original research papers, rapid communications and critical reviews in all aspects of fundamental and applied analytical chemistry.

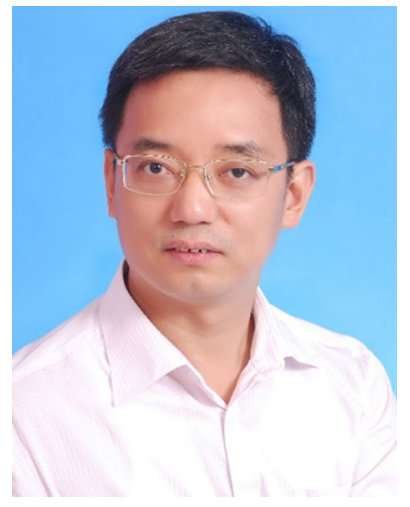

Professor Jin-Ming Lin graduated from Fuzhou University in 1984 and received a Ph.D. at Tokyo Metropolitan
University in 1997. He had studied and worked in Showa University and Tokyo Metropolitan University during 1992-2002. He was selected as "Hundred Talent Program" for Chinese Academy of Sciences, and obtained the Fund for Distinguished Young Scholars of National Natural Science Foundation of China at 2001. He had been a full professor in Research Center for Eco-Environmental Sciences, Chinese Academy of Science during 2002-2004. He is currently a full professor in Department of Chemistry, Tsinghua University since 2004, and was selected as Cheung Kung Scholars Professor of Ministry of Education, China at 2008. He is a Fellow of Royal Chemical Society, a vice president of Chinese Society of Mass Spectrometry, a General Secretary and deputy director of the Committee of Mass Spectrometry in Chinese Chemical Society, and service as associate editors or editorial boards for ten international journals. He is the author and co-author of 418 original research papers published in international journals, 45 reviews, 4 books and 48 patents.

His current research is focused on cell analysis, microfluidics with mass spectrometry (Chip-MS), and chemiluminescence. 